\title{
Chiral Metal Surfaces and Nanoparticles
}

\author{
Cyrille Gautier and Thomas Bürgi*
}

\begin{abstract}
The surface of metals can exhibit intrinsic chiral structure. Furthermore, chirality can be bestowed onto achiral metal surfaces by adsorption of chiral molecules. Such chiral metal surfaces are promising as heterogeneous enantioselective catalysts and may furthermore be used for the separation and detection of enantiomers. Similarly, metal nanoparticles can be chiral, which is reflected by their optical activity in metal-based electronic transitions. The transfer of chirality from adsorbate to the metal surface depends on the structure of the former, which is however difficult to elucidate. It is shown that vibrational circular dichroism can be used to determine the structure of a chiral adsorbed molecule and the way it interacts with the metal.
\end{abstract}

Keywords: Enantioselectivity · Nanoparticles · Optical activity · Surfaces · Vibrational circular dichroism

\section{Chiral Metal Surfaces}

Chemists tend to associate chirality with organic molecules, inorganic salts and biological materials, not necessarily with metals and surfaces. However, metal surfaces can indeed be chiral as well[1-6] and such surfaces have potential applications as heterogeneous enantioselective catalysts ${ }^{[7]}$ as well as for the separation and detection of enantiomers. ${ }^{[8-10]}$ The chirality of metal surfaces can have different natures as shown schematically in Fig.1. One possibility emerges from the fact that metal surfaces are not perfectly symmetric structures. They contain defects such as kinks, some of which may be chiral. Such intrinsically chiral sites can be created deliberately by cutting a metal single crystal along certain high Miller index planes. ${ }^{[1]}$ Fig. 1a shows a model of such a surface, in this case a (643) surface of a face-centred cubic metal. Note that on such a single crystalline surface all the chiral kink sites have the same absolute configuration. On a 'real' sample, i.e. a polycrystalline surface prepared for example by deposition of a metal layer, such chiral kinks may also exist. However, in the absence of additional chiral information a racemic mixture of left- and right-handed kink sites is expected in this case.

Apart from intrinsically chiral metal surfaces chirality can also be imparted onto achiral flat metal surfaces (terraces)

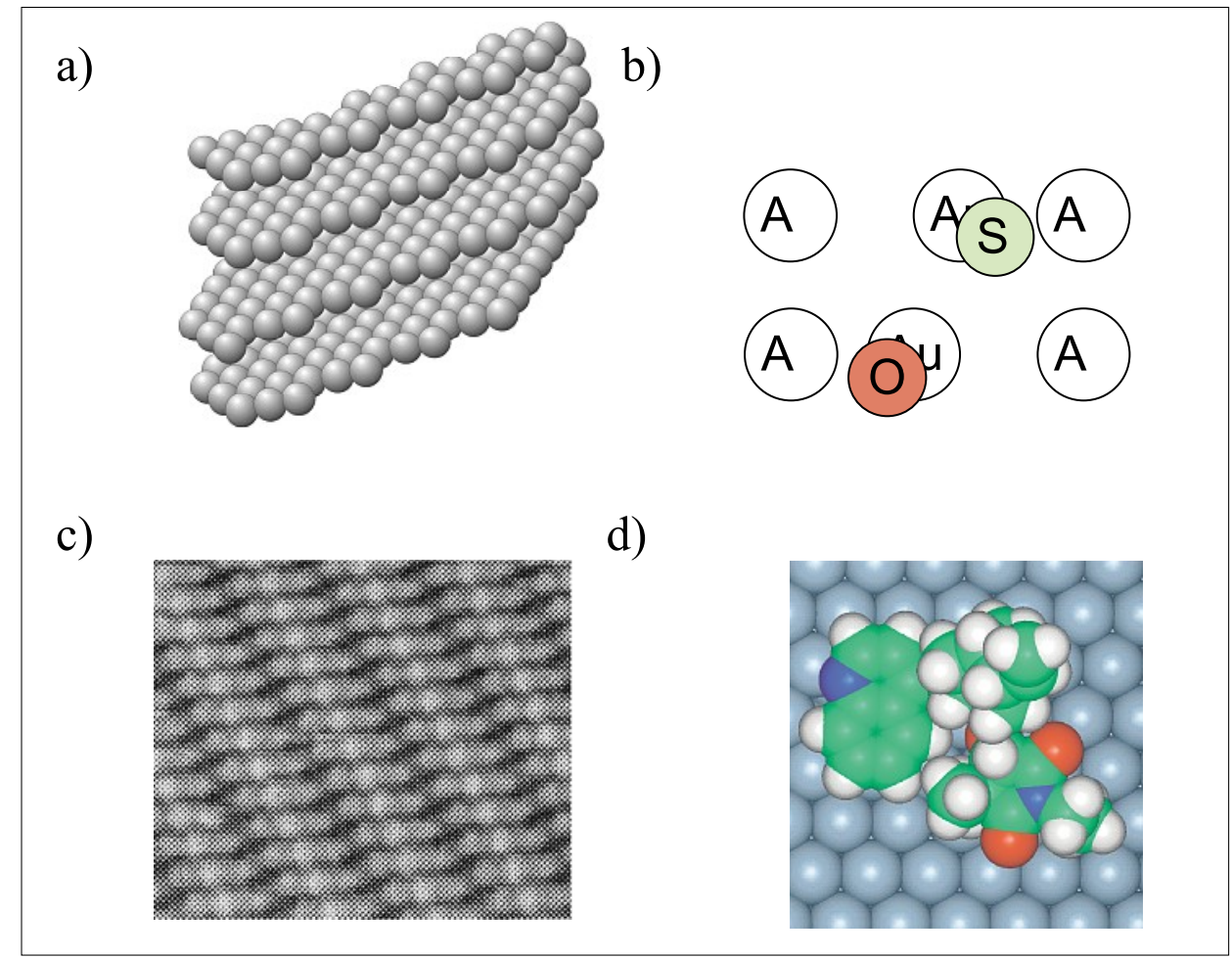

Fig. 1. Possibilities for chiral metal surfaces: a) Intrinsically chiral metal surfaces: Kink sites on a high Miller index single crystal surface. b) Model of inducing a chiral 'footprint' on a gold surface. The adsorbate interacts with the gold in a two point interaction via sulfur and oxygen atoms (thiolate and carboxylate, for example). This leads to a chiral distortion of the gold surface atoms. c) Supramolecular chirality on non-chiral terraces by self-assembly of chiral molecules. The example shows adsorbed tartaric acid on $\mathrm{Cu}(110)$. The adsorption pattern destroys all symmetry planes of the underlying metal surface. Such adsorption patterns may leave shaped assemblies of surface atoms free (template). d) A chiral site on a metal surface can be generated by adsorption of a chiral molecule. Part c) reprinted with permission from [4].

\footnotetext{
${ }^{\star}$ Correspondence: Prof. Dr. T. Bürgi

Institut de Microtechnique

Université de Neuchâte

CH-2009 Neuchâtel

Tel.: +41327182412

Fax: +41327182511

E-mail: thomas.burgi@unine.ch
} 
through adsorption of a chiral molecule. Several possibilities have been reported. For example, it has been shown that adsorption of large molecules may lead to a chiral reconstruction of the metal surface through the generation of chiral kink sites. ${ }^{[11]}$ The driving force for such a process is the larger adsorption energy of the molecule on the reconstructed surface with respect to adsorption on the non-reconstructed surface, which overcompensates the energy for reconstruction. A milder version of such a reconstruction is schematically shown in Fig. 1b. Upon adsorption of a chiral molecule, the metal surface atoms (gold in this case) in its neighbourhood are slightly displaced from their equilibrium position due to the local stress imposed by the molecule. Such a 'chiral footprint' has for example been proposed for the adsorption of tartaric acid on $\mathrm{Ni}(110) .{ }^{[12]}$

Finally, there is the possibility that the chirality of the metal surface is exclusively associated with the adsorbate layer on an otherwise achiral metal surface. Within this possibility one can distinguish between supramolecular chirality (Fig. 1c) and local chirality associated with individual adsorbed molecules (Fig. 1d). In the former case the molecule forms long-range ordered patterns on the metal surface, in such a way that the symmetry planes of the underlying metal surface are destroyed. The example in Fig. 1c represents the case of tartaric acid on $\mathrm{Cu}(110) .{ }^{[13]}$ Generation of chiral sites on metal surfaces by adsorption of chiral molecules (Fig. 1d) is a promising strategy for the preparation of heterogeneous enantioselective catalysts. ${ }^{[14-16]}$ In this case the catalytic activity of the metal (e.g. for hydrogenation) is combined with the chiral information associated with the adsorbate.

The examples provided above clearly demonstrate that chirality can be bestowed to metal surfaces and this opens promising possibilities for applications in various fields of chiral technology. In the last ten years surface scientists became more and more interested in fundamental and applied aspects of chiral surfaces. In a further step we were interested to impart chirality to metal nanoparticles. Small metal particles consist almost of surface only. For example, for a spherical metal particle with a core diameter of $2 \mathrm{~nm}$ about $40 \%$ of the metal atoms reside at the surface. In this article we focus on gold nanoparticles stabilized with chiral thiols, which can be viewed as the nanometer-sized analogues of self-assembled monolayers (SAMs) of thiols on flat gold surfaces.[17] The preparation of gold nanoparticles covered with thiols is well established ${ }^{[18]}$ and it is currently an extremely active field of research due to the promising properties of these nanomaterials. Gold nanoparticles covered by chiral thiols may exhibit chiral structures analogous to those

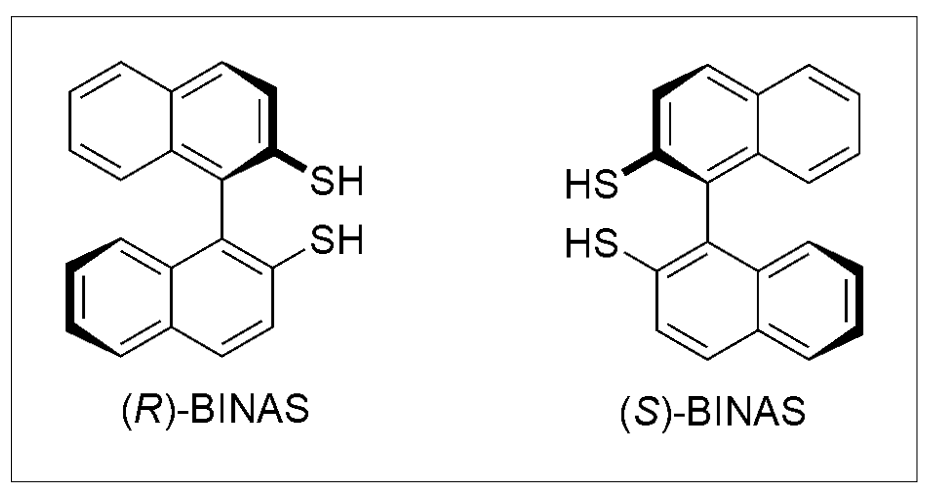

Fig. 2. Structure of $(R)-1,1^{\prime}$-binaphthyl-2,2'-dithiol [(R)-BINAS] and (S)1,1'-binaphthyl-2,2'-dithiol [(S)-BINAS]

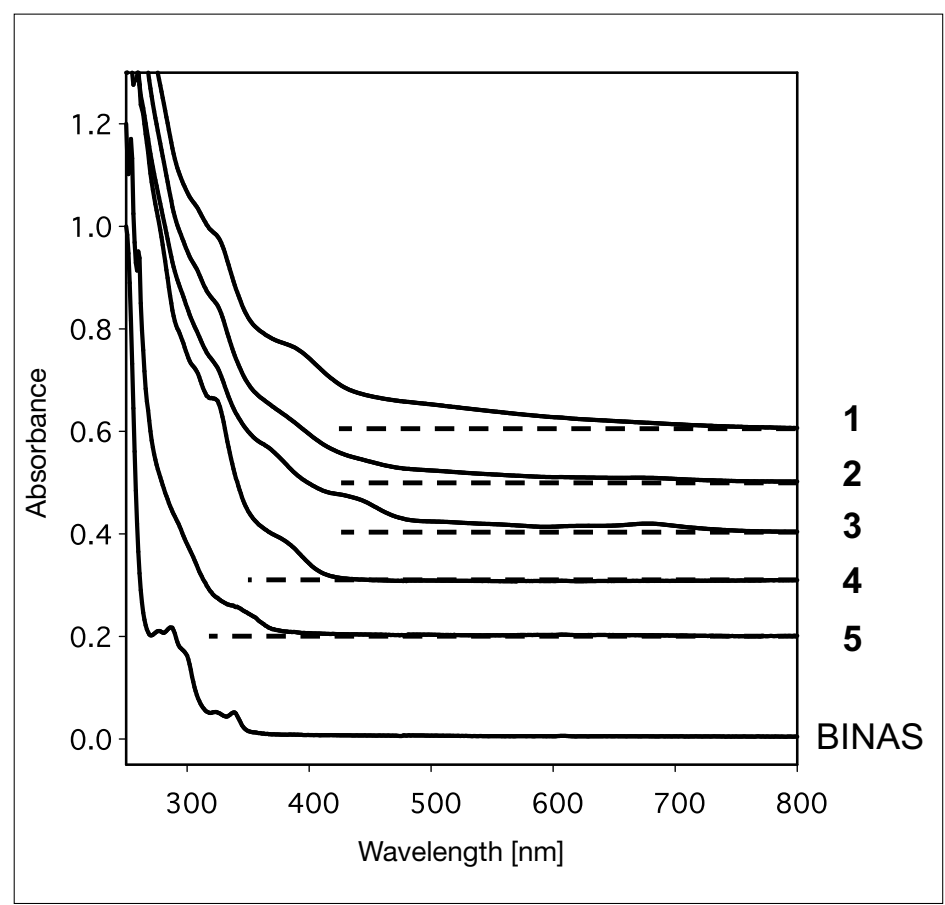

Fig. 3. UV-vis spectra of separated fractions $\mathbf{1 - 5}$ of gold particles covered with BINAS and of BINAS. The UV-vis spectra were scaled to 1 absorbance unit at $250 \mathrm{~nm}$ and offset for clarity. Adapted with permission from [20].

discussed above (Fig. 1) for the flat metal surfaces. However, the investigation of flat surfaces and nanoparticles relies on rather different techniques. Whereas the investigation of chiral metal surfaces largely relies on scanning tunneling microscopy (STM), the solubility of nanoparticles allows the application of chiroptical techniques, such as circular dichroism (CD) and vibrational circular dichroism (VCD), which specifically probe chirality. The former technique is sensitive to electronic transitions that might be located in the metal core, whereas the latter technique probes molecular vibrations and is therefore sensitive to the adsorbed molecules.

\section{Chiral Metal Particles}

Small gold nanoparticles covered by thiolate ligands can be prepared by addition of the corresponding thiol to a $\mathrm{Au}$ (III) solution followed by reduction by $\mathrm{NaBH}_{4}$. For non water-soluble thiols a two-phase method can be applied ${ }^{[19]}$ by using a phase transfer agent. By such a method gold particles covered by $(R)$-1,1'-binaphthyl-2,2'-dithiol $((R)$-BINAS) and $(S)$-1,1'-binaphthyl-2,2'dithiol $((S)$-BINAS) were prepared (Fig. $2)^{[20]}$ Transmission electron microscopy (TEM) reveals that the particles are smaller than $3 \mathrm{~nm}$. During the chemical reduction method outlined above the gold nanoparticles are formed by nucleation of metallic gold atoms, growth of the particles and passivation by the thiols, which inevitably leads to particles with different sizes, i.e. to a size distribution. Using size exclusion chromatography (SEC) it was possible to separate different sizes of particles. In total five fractions with different color were obtained. The absorption spectra of the different fractions $\mathbf{1} \mathbf{- 5}$ shown in Fig. 3 clearly 
depend on the size of the particles ( 1 denoting the largest size fraction) and show considerable structure, which shows that the separated fractions contain monodisperse particles. Also, the absorption spectra are distinctly different from the ones of BINAS. There is a clear trend in the spectra as concerns the absorption onset, which shifts to longer wavelengths with increasing particle size. This trend was already observed for gold particles covered by glutathione ${ }^{[21,22]}$ and $\mathrm{N}$-isobutyryl-cysteine, ${ }^{[23]}$ respectively, separated into different compounds by gel electrophoresis.

Fig. 4 shows the $\mathrm{CD}$ spectra of some separated fractions. The size exclusion column is shown as well. The CD spectra of the gold particles covered by the enantiomers of BINAS show a mirror image relationship and are distinctly different from the CD spectrum of the free BINAS. Therefore, the optical activity observed for the gold particles covered by the BINAS is associated with electronic transitions within the metal core. Similar to the UV-vis spectra the CD spectra depend on the particle size. This is particularly apparent from a comparison between fractions 3 and $\mathbf{4}$. It should be noted that the CD spectra are distinctly different from the ones reported for small gold particles covered by the water-soluble thiols glutathione, ${ }^{[21]}$ penicillamine ${ }^{[24]}$ and $\mathrm{N}$-isobutyryl-cysteine, ${ }^{[25]}$ respectively.

There are only very few reports of optically active and size-separated metal nanoparticles and the knowledge gained up to now can be summarized as follows: Optical activity differs with the size of the NPs and generally decreases as the size increases. As for classical chiral molecules, the circular dichroism (CD) spectra of NPs display a mirror image relationship when covered with the two opposite enantiomers and no optical activity is observed when a racemic mixture of ligand is used. A common feature of the modifiers inducing CD signals reported up to now is that they contain several functional groups able to coordinate to the gold particle.

The observation of optical activity in metal-based electronic transitions shows that the electronic structure of the metal core is chiral. The key question is whether the metal core is intrinsically chiral or whether the optical activity is induced onto an achiral metal core. Usually, the structure of NPs is assumed to correspond to a fragment of the highly symmetric bulk crystal lattice. From a fundamental point of view, however, the very small particles investigated here (a few tens of metal atoms only) can be understood as the intermediate state of matter between a single metal atom and the bulk metal and a structural evolution with the size of the particles can be expected. Indeed, in 1996 calculations performed by Wetzel and DePristo, and experimental ob-

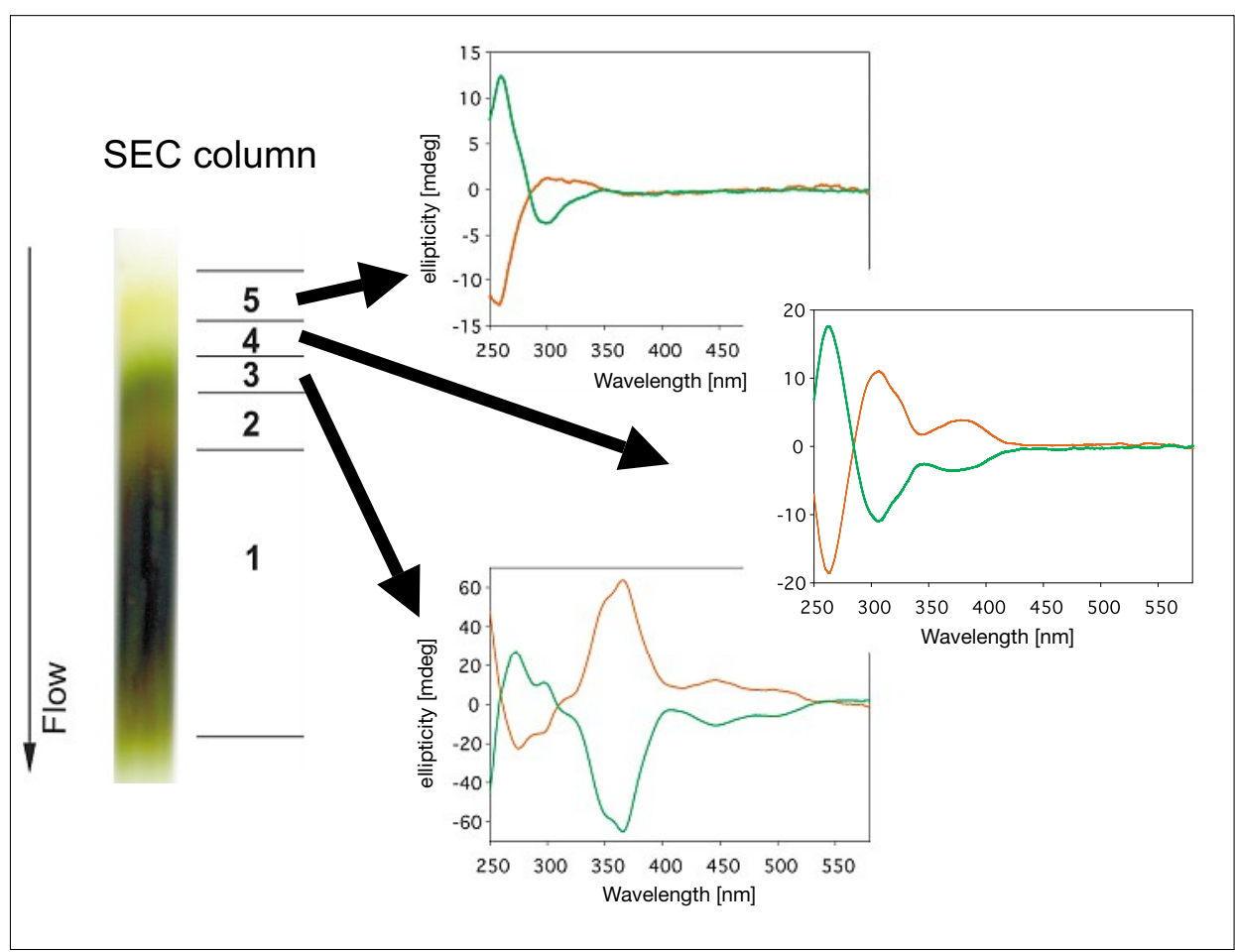

Fig. 4. Left: Size exclusion chromatography separation of BINAS protected gold particles. The separated fractions are numbered from 1-5 according to their decreasing elution mobility. Horizontal lines show roughly the limit between adjacent fractions. Right: CD spectra of separated fractions 3-5 of gold particles covered with (S)-BINAS (green) and $(R)$-BINAS (red). Adapted with permission from [20].

servation of Riley et al. indicated that naked $\mathrm{Ni}_{39}$ clusters prefer a lower $\left(D_{5}\right)$ symmetry, i.e. a chiral structure. ${ }^{[26,27]}$

The observed optical activity in metal-based electronic transitions can be explained in different ways. It could arise from an intrinsically chiral inorganic core. In the presence of adsorbed chiral ligands diastereomers are formed and one of the two possible enantiomers of the core is favored. Alternatively, the inorganic core could be achiral and the optical activity could be induced by a chiral environment due to the chiral organic shell through a vicinal effect or through a chiral electrostatic field. Both models have support from theory. Garzón et al. have predicted that small metal particles such as $\mathrm{Au}_{28}$ or $\mathrm{Au}_{55}$ prefer low symmetry chiral over high-symmetry nonchiral structures. ${ }^{[28,29]}$ Goldsmith and co-workers have demonstrated that optical activity could arise from an achiral metal core perturbated by a dissymmetric field originating from the chiral organic shell. ${ }^{[30]}$ Trends in the electronic transition frequencies and amplitudes with cluster size observed experimentally are qualitatively in agreement with these two models. In an intermediate model, the grand core can be achiral but the relaxation of the surface atoms involved in the adsorption of the chiral ligand creates a chiral 'footprint' on the particle surface similar to that proposed for tartaric acid adsorption on Ni surfaces. ${ }^{[12]}$ Such a model is supported by the recently presented first total structure determination by X-ray crystallography of a small goldthiolate nanocluster composed of 102 gold atoms and $44 p$-mercaptobenzoic acids ( $p$ MBA) ${ }^{[31]}$ The particles are chiral although $p$-MPA is not and crystallized with half of an enantiomer in the asymmetric unit of the crystal. However, the central gold atoms are packed in a Marks decahedron, which is highly symmetric as the fcc structure of bulk gold (Fig. 5). The chirality of the particles (gold core plus ligands) arises at two levels. First of all, the thiolates form staple structures with a gold atom located between two sulfur atoms (Fig. 5b). In these structures the sulfur atoms represent stereogenic centers. Second, the arrangement of the equatorial surface atoms is chiral (Fig. 5a). The deviations in local symmetry may reflect the interaction of the equatorial atoms with the thiolate layer. It is in an analogous manner that a chiral thiol may induce a chiral and enantiopure arrangement of the gold surface atoms, which leads to the observed optical activity in metal-based electronic transitions. It is then clear that the structure of the adsorbed thiolate and the way it interacts with the gold particle surface plays a crucial role for the chiral reconstruction ('footprint') on the particle. However, such structural information is difficult to obtain and suitable crystals for X-ray structural analysis are rarely obtained for nanoparticles. In the following it is shown that vibrational circular dichroism (VCD) is a 


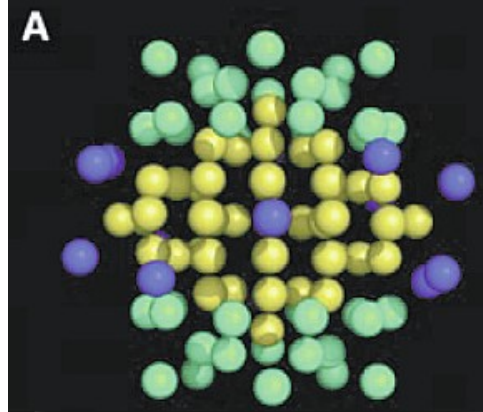

$\mathbf{B}$

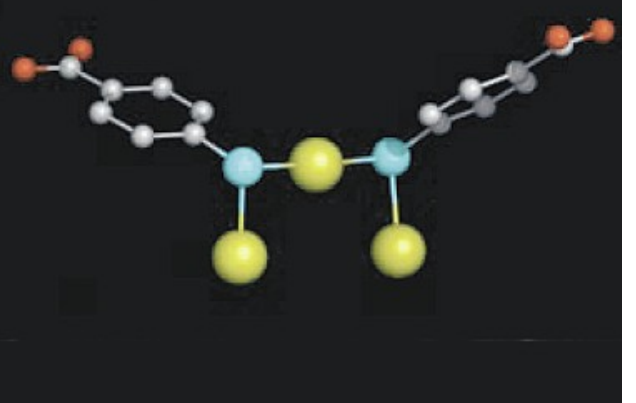

Fig. 5. X-ray crystal structure determination of the $\mathrm{Au}_{102}(\mathrm{p}-\mathrm{MBA})_{44}$ nanoparticle. (A) Packing of gold atoms in the core. Marks decahedron in yellow, two 20-atom 'caps' at the poles in green, and the 13-atom equatorial band in blue. (B) Sulfur-gold interactions in the surface of the nanoparticle. Example of two $p$-MBAs interacting with three gold atoms in a bridge conformation (staple). Gold atoms are yellow, sulfur atoms are cyan, oxygen atoms are red, and carbon atoms are gray. Adapted with permission from [31].
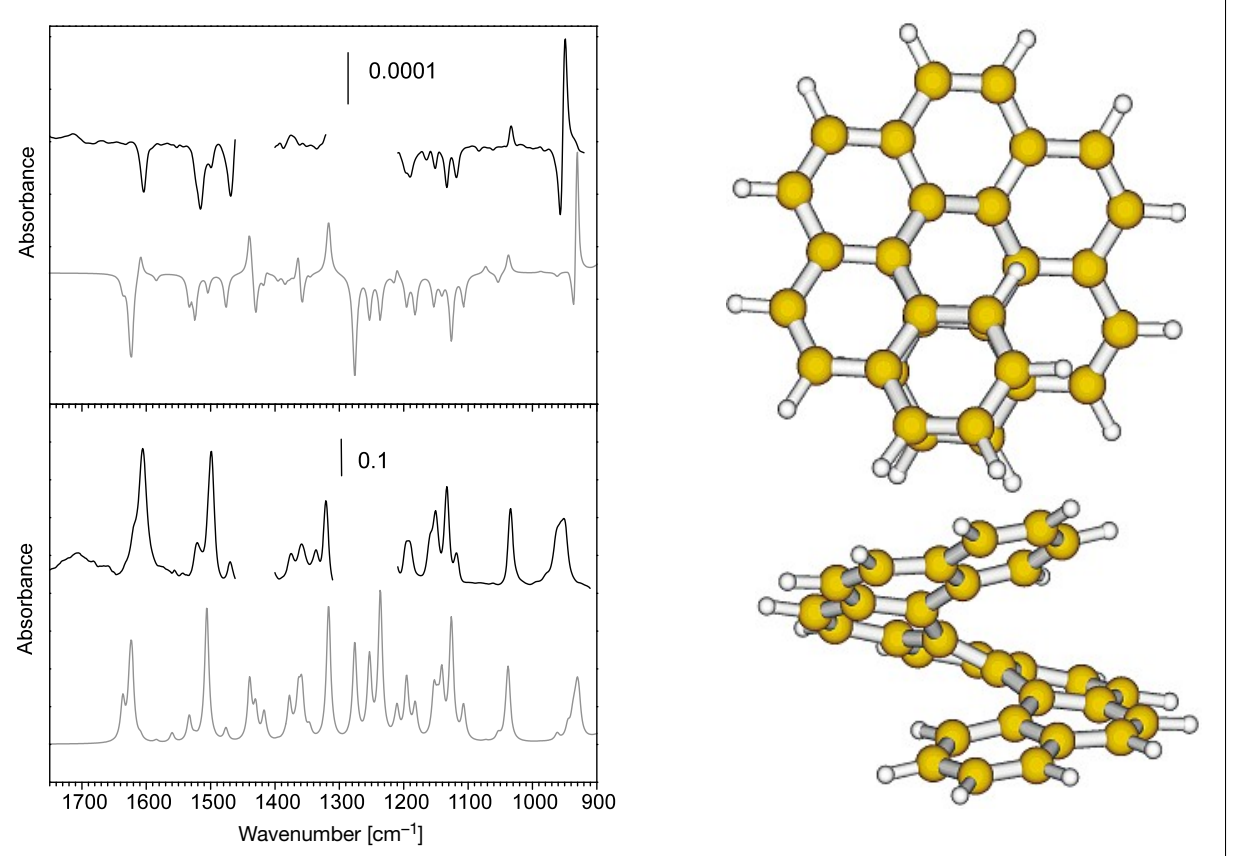

Fig. 6. Comparison between calculated (grey) and experimental (black) IR (bottom) and VCD spectra of heptahelixene. The experimental spectra were recorded in $\mathrm{CH}_{2} \mathrm{Cl}_{2}$ by averaging 3600 scans. The calculations were performed at the B3LYP / 6-31G $(d, p)$ level of theory on the M enantiomer shown in the Figure. Adapted with permission from [43].

powerful tool, not only to obtain structural information on chiral molecules, but also on chiral adsorbates on small gold particles.

\section{Vibrational Circular Dichroism}

Vibrational circular dichroism (VCD) is the differential absorption of left and right circularly polarized infrared light. ${ }^{[32]}$ Anisotropy factors are usually small, $\Delta \mathrm{A} / \mathrm{A}$ of $10^{-4}$ to $10^{-5}$ is typical, where $\mathrm{A}$ is the absorbance and $\Delta \mathrm{A}$ is the difference in absorbance for left- and right-circularly polarized light. With technological developments such as lock-in amplifiers, liquid-nitrogen- cooled semiconductor detectors and IR polarization modulators for the generation of left- and right-circularly polarized radiation the measurement of such small signals is nowadays possible. [33] Still, the measurement of good quality spectra for 'challenging' samples can easily take hours.

VCD spectra contain substantial structural information. First of all, the technique can be used to determine the absolute configuration of a sample without the need to grow single crystals. ${ }^{[34-37]}$ Second, and perhaps even more important, the technique yields information on the conformation of dissolved molecules. ${ }^{[38-41]}$ This information has to be extracted from the experimental spectrum through comparison with theory. For this the possible conformations of the molecule have to be determined, their structure refined and their VCD spectrum calculated. Generally the VCD spectra of different conformers differ significantly. This strategy for structure determination of dissolved molecules obviously relies on the quality of the calculation. Today methods are available, notably relying on density functional theory (DFT), which have predictive character for VCD spectra. ${ }^{[42]}$ This is illustrated in Fig. 6, which shows the comparison between measured and calculated IR and VCD spectra of heptahelicene. ${ }^{43]}$ As is obvious, good qualitative agreement between experimental and theoretical spectra can be obtained despite the approximations inherent in the calculations, such as harmonic approximation for the vibrational analysis and neglect, in this case, of solvent.

Fig. 7 demonstrates the potential of VCD spectroscopy to probe the interaction between chiral and non-chiral molecules in solution. ${ }^{[41]}$ As an example the interaction between the alkaloids cinchonidine and the near enantiomer cinchonine, respectively, and trifluoroacetic acid is studied. Fig. 7 (top) shows the proposed structure of a 1:1 acid-alkaloid complex. The Fig. also shows infrared and VCD spectra of equimolar solutions of the alkaloids and trifluoroacetic acid. The dominant band in the spectra is associated with the antisymmetric carboxylate vibration, which is localized on the non-chiral molecule. Still, the spectra show that this mode exhibits VCD activity. Through its bonding to the chiral alkaloid VCD activity is induced in the non-chiral acid. Therefore the active vibration on the acid probes the chiral site to which it is bound. Although the two alkaloids are diastereomers (they differ in the position of the vinyl group) the sites where the acid is bound are to a good approximation mirror images. As a consequence the induced vibrational optical activity of the carboxylate vibration has opposite sign in the two cases. This also emerges from the calculations (B3LYP/6-31G*), which show rotational strengths for this vibration of $-460 \times 10^{-44} \mathrm{esu}^{2} \mathrm{~cm}^{2}$ for the complex with cinchonidine and $586 \times 10^{-44} \mathrm{esu}^{2} \mathrm{~cm}^{2}$ for the corresponding one with cinchonine. The examples outlined above show the potential of VCD spectroscopy to study chirality at the molecular level.

\section{Vibrational Circular Dichroism of Gold Nanoparticles}

We have recently applied VCD spectroscopy to nanoparticles for the first time in order to study the conformation of the adsorbed chiral molecules. ${ }^{[23,44]}$ The cysteine 
derivatives $\mathrm{N}$-acetyl-cysteine and $\mathrm{N}$-isobutyryl-cysteine adsorbed on gold NPs of about $2 \mathrm{~nm}$ core diameter were studied. VCD spectra of gold NPs covered by the two enantiomers of $\mathrm{N}$-isobutyryl-cysteine (NIC) were measured. ${ }^{[23]}$ Whereas the IR spectra were identical for the two enantiomers, the VCD spectra showed mirror image relationship. Different conformers and different adsorption modes of the molecule adsorbed on a small gold cluster were considered for the calculation. Density functional theory (DFT) calculations also showed that the structure of the underlying gold cluster does not have a large effect on the simulated VCD spectra whereas the conformation and the adsorption mode of the thiolate have a large influence. The simulated VCD spectrum of a stable calculated conformer for $\mathrm{N}$-isobutyryl-cysteine adsorbed on a small gold cluster matches reasonably well with the experimental data, as shown in Fig. 8. ${ }^{[23]}$ This conformation is characterized by an interaction of the carboxylate with the gold particle. Thus the carboxylate group seems to be a second anchoring point beside the strong $\mathrm{Au}-\mathrm{S}$ bond. The structure is in agreement with independent orientation measurements of $\mathrm{N}$-acetylcysteine on gold surfaces ${ }^{[45]}$ investigated by attenuated total reflection infrared spectroscopy (ATR-IR). ${ }^{[46]}$ This two-point interaction may influence the optical activity in metal-based transitions, which are actually quite pronounced for these particles, ${ }^{[23]}$ because such a two-point interaction may help imparting chirality to the metal.

Most importantly, it has been shown that VCD is a promising tool to study the structure of adsorbed chiral molecules on small metal nanoparticles. Such information is relevant because the structure (conformation) of the adsorbed molecules can have a pronounced effect on surface properties and function such as molecular recognition and heterogeneous enantioselective catalysis. It should be noted that the two nanoparticle systems investigated up to now by VCD spectroscopy (gold$\mathrm{N}$-acetyl-cysteine and gold-N-isobutyrylcysteine) are challenging for mainly two reasons. First of all N-acetyl-cysteine and $\mathrm{N}$-isobutyryl-cysteine are quite flexible molecules with considerable conformational freedom. Therefore, several conformers might contribute to the experimental spectrum. Second, the particles that were studied up to now are soluble in water. It is very likely that water specifically interacts with $\mathrm{N}$-acetyl-cysteine and $\mathrm{N}$-isobutyryl-cysteine via hydrogen bonding and this will influence the VCD at least to a certain extent. This should be included in the calculation of the VCD spectra. Taking into account this potential for improvement the approach for studying the structure of adsorbed chiral molecules on metal

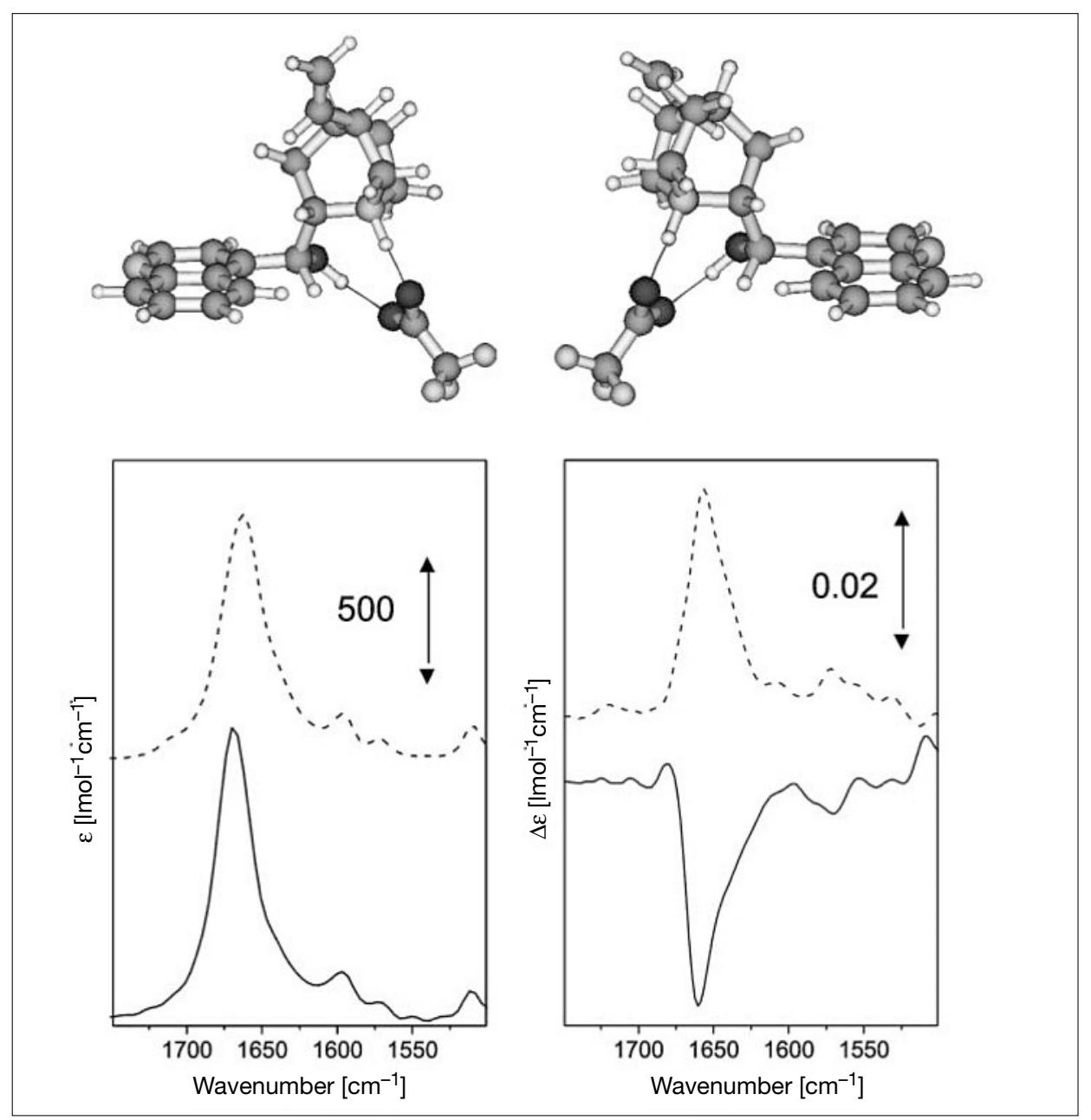

Fig. 7. Calculated structures of complexes between cinchonidine (left) and cinchonine (right), respectively, and trifluoroacetic acid. Lines represent hydrogen-bonding interactions. Bottom: Infrared (left) and VCD spectra (right) of $1: 1$ mixtures $(0.02 \mathrm{~mol} / \mathrm{l})$ of cinchonidine (solid line) and cinchonine (dashed line), respectively, and trifluoroacetic acid in $\mathrm{CDCl}_{3}$. Reprinted with permission from [41].

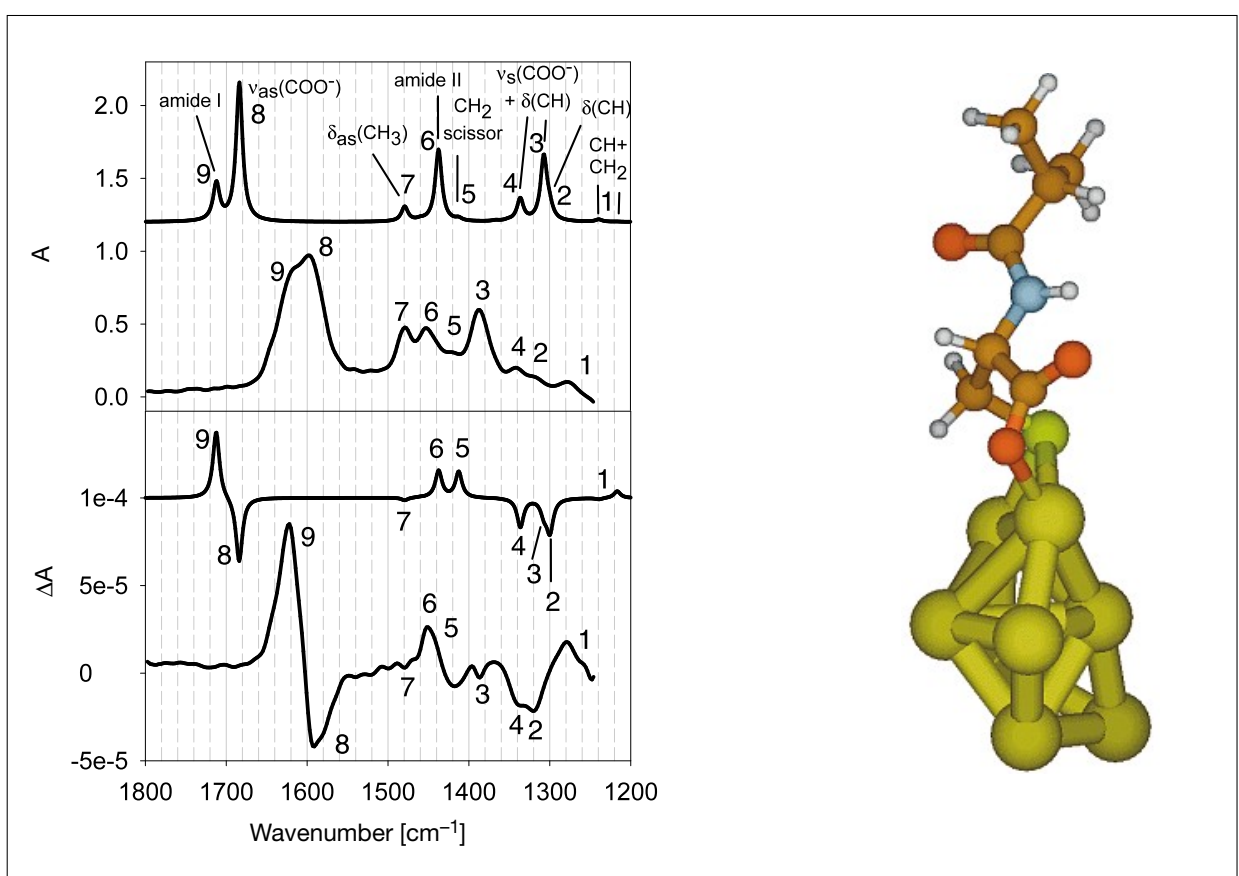

Fig. 8. Comparison between calculated and experimental IR and VCD spectra of N-isobutyryl-Lcysteine on gold nanoparticles (left). The calculated spectra are shown for the conformer on the right. Corresponding bands are numbered and the assignment is given in the upper part of the Figure. The experimental spectra were recorded for gold nanoparticles covered by $\mathrm{N}$-isobutyryl-L-cysteine dissolved in $\mathrm{D}_{2} \mathrm{O}$. Reprinted with permission from [23]. Copyright (2005) American Chemical Society. 
particles outlined above can be considered very promising.

\section{Acknowledgement}

The authors thank the Swiss National Science Foundation for financial support and the Swiss National Supercomputing Centre for grants of computing time.

Received: March 3, 2008

[1] C. F. McFadden, P. S. Cremer, A. J. Gellman, Langmuir 1996, 12, 2483.

[2] G. A. Attard, J. Phys. Chem. B 2001, 105, 3158.

[3] D. Ferri, T. Bürgi, J. Am. Chem. Soc. 2001, $123,12074$.

[4] M. O. Lorenzo, C. J. Baddeley, C. Muryn, R. Raval, Nature 2000, 404, 376.

[5] M. Schunack, E. Laegsgaard, I. Steengaard, I. Johannsen, F. Besenbacher, Angew. Chem., Int. Ed. 2001, 40, 2623.

[6] R. Fasel, M. Parschau, K. H. Ernst, Nature 2006, 439, 449.

[7] T. Bürgi, A. Baiker, Acc. Chem. Res. 2004, 37, 909.

[8] T. Nakanishi, N. Yamakawa, T. Asahi, T. Osaka, B. Ohtani, K. Uosaki, J. Am. Chem. Soc. 2002, 124, 740.

[9] M. Bieri, T. Bürgi, J. Phys. Chem. B 2005, 109, 10243.

[10] M. Bieri, T. Bürgi, Chem. Phys. Chem. 2006, 7, 514.

[11] M. Schunack, L. Petersen, A. Kuhnle, E. Laegsgaard, I. Stensgaard, I. Johannsen, F. Besenbacher, Phys. Rev. Lett. 2000, 86, 456.

[12] V. Humblot, S. Haq, C. Muryn, W. A. Hofer, R. Raval, J. Am. Chem. Soc. 2002, 124, 503.

[13] M. O. Lorenzo, S. Haq, T. Bertrams, P. Murray, R. Raval, C. J. Baddeley, J. Phys. Chem. B 1999, 103, 10661.
[14] T. Bürgi, A. Baiker, J. Phys. Chem. B 2002, 106, 10649.

[15] D. Ferri, T. Bürgi, A. Baiker, J. Chem. Soc. Chem. Commun. 2001, 1172.

[16] D. Ferri, T. Bürgi, A. Baiker, J. Catal. 2002, 210, 160.

[17] A. Ulman, Chem. Rev. 1996, 96, 1533.

[18] M.-C. Daniel, D. Astruc, Chem. Rev. 2004, 104, 293.

[19] M. Brust, M. Walker, D. Bethell, D. J. Schiffrin, R. Whyman, J. Chem. Soc. Chem. Commun. 1994, 801.

[20] C. Gautier, R. Taras, S. Gladiali, T. Bürgi, Chirality 2008, 20, 486.

[21] T. G. Schaaff, R. L. Whetten, J. Phys. Chem. B 2000, 104, 2630.

[22] Y. Negishi, K. Nobusada, T. Tsukuda, J. Am. Chem. Soc. 2005, 127, 5261.

[23] C. Gautier, T. Bürgi, J. Am. Chem. Soc. 2006, 128, 11079.

[24] H. Yao, K. Miki, N. Nishida, A. Sasaki, K. Kimura, J. Am. Chem. Soc. 2005, 127 , 15536.

[25] M. Bieri, C. Gautier, T. Bürgi, Phys. Chem. Chem. Phys. 2006, 9, 671.

[26] T. L. Wetzel, A. E. DePristo, J. Chem. Phys. 1996, 105, 572.

[27] E. K. Parks, K. P. Kerns, S. J. Riley, J. Chem. Phys. 1998, 109, 10207.

[28] I. L. Garzon, M. R. Beltran, C. Gonzalez, I. Gutierrez-Gonzalez, K. Michaelian, J. A. Reyes-Nava, J. I. Rodriguez-Hernandez, Eur. Phys. J. D 2003, 24, 105.

[29] I. L. Garzon, J. A. Reyes-Nava, J. I. Rodriguez-Hernandez, I. Sigal, M. R. Beltran, K. Michaelian, Phys. Rev. B 2002, 66, 73403.

[30] M.-R. Goldsmith, C. B. George, G. Zuber, R. Naaman, D. H. Waldeck, P. Wipf, D. N. Beratan, Phys. Chem. Chem. Phys. 2006 8, 63 .

[31] P. D. Jadzinsky, G. Calero, C. J. Ackerman, D. A. Bushnell, R. D. Kornberg, Science 2007, 318, 430.
[32] L.A. Nafie, T. A. Keiderling, P. J. Stephens, J. Am. Chem. Soc. 1976, 98, 2715.

[33] L. A. Nafie, Annu. Rev. Phys. Chem. 1997, $48,357$.

[34] T. B. Freedman, X. Cao, R. K. Dukor, L. A. Nafie, Chirality 2003, 15, 743.

[35] C. Herse, D. Bas, F. C. Krebs, T. Bürgi, J. Weber, T. Wesolowski, B. W. Laursen, J. Lacour, Angew. Chem., Int. Ed. 2003, 42, 3162.

[36] D. Bas, T. Bürgi, J. Lacour, J. Vachon, J. Weber, Chirality 2004, 17, S143.

[37] J. V. Naubron, L. Giordano, F. Fotiadu, T. Bürgi, N. Vanthuyne, C. Russel, J. Org. Chem. 2006, 71, 5586.

[38] C. N. Su, T. A. Keiderling, J. Am. Chem. Soc. 1980, 102, 511.

[39] R. Schweitzer-Stenner, F. Eker, K. Griebenow, X. L. Cao, L. A. Nafie, J. Am. Chem. Soc. 2004, 126, 2768.

[40] P. Bour, H. Navratilova, V. Setnicka, M. Urbanova, K. Volka, J. Org. Chem. 2002, 67, 161.

[41] T. Bürgi, A. Vargas, A. Baiker, J. Chem. Soc. Perkin Trans. 2 2002, 1596.

[42] F. J. Devlin, P. J. Stephens, J. R. Cheeseman, M. J. Frisch, J. Phys. Chem. A 1997, 101, 9912.

[43] T. Bürgi, U. Urakawa, B. Behzadi, K.-H. Ernst, A. Baiker, New. J. Chem. 2004, 28 , 332.

[44] C. Gautier, T. Bürgi, Chem. Commun. 2005, 5393.

[45] M. Bieri, T. Bürgi, J. Phys. Chem. B 2005, 109, 22476.

[46] T. Bürgi, A. Baiker, Adv. Catal. 2006, 50, 228. 